however, partly as a result of Hopkins's own valuable researches, and it is to be hoped that this book may hasten the progress by interesting African nationals in the subject. Conservation of ratural vegetation can only be achieved effectively by those who live in the country concerned having an intelligent appreciation of the need for it. Forest and Savanna should have a part to play in this all-important educational aspect. In particular the fifth chapter, which doals with the relationship between forest and savanna, brings out many points concerning land management that are vital to the inter-related aspects of agriculture, forestry and conservation. Two appendixes, dealing with field methods for vegetation analysis and suggested student projects, will be of special value to the instructor. References and an index conclude this small text-book which should rapidly find an ecological niche in the curricula of school sixth-forms and first-year university courses.

F. N. Hepper

\section{Mathematical Analysis}

Differentiation and Integration. By I. G. Aramanovich, R. S. Guter, L. A. Lyusternik, I. L. Raukhvarger, M. I. Skanavi and A. R. Yanpol'skii. Translated by H. Moss. English edition edited by I. N. Sneddon. (International Series of Monographs in Pure and Applied Mathematies, Vol. 81.) Pp. xi +322. (London and New York: Pergamon Press, Ltd., 1965.) 63s. net.

ThIs book contains a concise account of the properties of differentiation, differentials, Riemann integration in one and several dimensions, together with implicit function theory and the effect of transformations in differentiation and integration theory. The Stieltjes integral is briefly introduced.

There are no proofs, examples, or exorcises, but there are several counter-examples to show where and why the theory will not extend. This renders the book useful for reference for the non-specialist, and it might be valuable to a lecturer planning a course, but not to a student.

The fact that differont authors have written different parts is apparent in an inconsistent notation. Thus, for example, $y[n](x)$ and $y(n)(x)$ are used to denote the Schwartzian derivative, and elsewhere both $y(n)(x)$ and $y^{n}(x)$ denote the ordinary derivative. The book concludes with useful lists of integrals, etc., and a few tables of higher transcendental functions.

\section{R. L. Perry}

\section{Die Tierische Zelle in Zellkultur}

Von R. Schindler. (Fortschritte der Krobsforschung. Recent Results in Cancer Research, Vol. 1.) Pp. vi +88. (Berlin: Springor-Verlag, 1965.) 16 D.M.

To assess the value of cell culture in cancer research, it is clearly necessary to consider many different aspects. In the series on "Recent Results in Cancer Research", published by Springer, the author, Dr. R. Schindler, has discussed the culture of animal cells rather generally. This book deals with various eulture methods, basic medium and biochemical requirements of cells, pharmacological studies, cytogenetics, specific cell functions, the mitotic cycle, effects of ionizing radiation and changes brought about by viruses. The main description of cancer cells in culture is given in the last chapter. There is an extensive bibliography of more than 700 references. The book therefore provides good background reading and a good bibliography for people interested in taking up cell culture work on cancer prob. lems. A great deal of work has been done on cancer-cell culture-both hanging-drop cultures and cell lines in monolayer culture, and more recently in organ culture. One could wish that the author had covered these subjects in more detail. For the study of human cancer, where it is impossible to carry out experimental work in vivo, with one or two exceptions involving transplantation of tissues into animals. the main future is likely to lie in the use of cultures for experimental work.

\section{MEETINGS}

\section{GEOPHYSICS AND COMPUTERS}

The Department of Applied Mathematies and Theoretical Fhysics at Cambridge was host from June 27 to July 5 to the Third International Symposium on Geophysical Theory and Computers, an activity of the I.U.G.G. Upper Mantle Committee.

About eighty-five attended the symposium, and more than forty papers were given, each between half an hour and an hour in length. It would be impossible to mention every paper-invidious though it is to mention particular contributions, certain recurrent new themes emerged which are specially worthy of report.

The major problem, one might say the only problem, in geophysics is that of inversion. Given a set or sets of data, what can be deduced about continuum parameters of specified regions of the Earth? And is that information a sufficient guide to the composition and thermodynamies of the region? The question of uniqueness of interpretation has until recently been treated in a rather indefinite way in seismology. Whereas it is fairly easy to show that surface observations of gravity and magnetic fields are insufficient to give unique distributions of density and magnetization beneath the surface, the uniqueness or otherwise of seismic data (which seems likely to be the principal source of information about the deep interior of the Earth for some time) is much more difficult to establish.

Prof. V. I. Keilis-Borok and two colleagues from the U.S.S.R. gave summaries of the present situation with regard to body wave travel times, where uniqueness has been a pressing problem. It is demonstrable that in the presence of a 'Iow velocity layer', two different subsurface velocity distributions may yield the same travel time curve. However, the situation is in practice bedevilled by the difficulties of positively identifying body wave signals on actual seismograms. A lively debate between Miss I. Lehmann (Copenhagen) and Dr. E. A. Flinn (Alexandria, Virginia) on work which the latter had presented using array processing techniques epitomized this problem. However, as Flinn and others, notably Dr. D. H. Weichert (Ottawa), Dr. E. J. Kelly (Lexington, Massachusetts) and Dr. R. A. Phinney (Princeton) showed, array processing is becoming a valuable tool in mantle and core geophysics.

The uniqueness, or otherwise, of normal mode data (from free oscillations of the Earth, Rayleigh waves and dove waves) has been an equally thorny problem. A notable paper by Prof. G. E. Backus and Prof. F. Gilbert (La Jolla, California) showed, among other things, that more than one density distribution can satisfy the same data to any prescribed degree of accuracy. This paper, with the Russian contributions, clearly showed the necessity of developing further criteria for the acceptanee of Earth models.

One such consideration is scismic amplitude data, and much more reliable information about amplitude as a function of range from source is being compiled by the U.K. Atomic Energy Authority group, and was presented by Dr. A. Douglas (Aldermaston).

Another consideration is the plausibility of an Earth model on solid-state criteria. Prof. D. L. Anderson (California Institute of 'Technology) indicated the questions which we would wish to ask of solid-state physics, and Prof. L. Knopoff (University of California, Los Angeles) indicated the possible uses of solid-state physics in combination with seismology in the determination of reasonable composition models.

A seismological parameter of increasing importance and whose significance is still not fully understood is dissipation. There is no doubt that this too will have to be taken into account in any unified view of the Earth's structure. 